\title{
Josephson junctions and dark energy
}

\author{
Philippe Jetzer and Norbert Straumann \\ Institute for Theoretical Physics University of Zurich, \\ Winterthurerstrasse 190, \\ CH-8057 Zurich, Switzerland
}

October 23, 2018

\begin{abstract}
In a recent paper Beck and Mackey astro-ph/0603397 argue that the argument we gave in our paper [Phys. Lett. B 606, 77 (2005)] to disprove their claim that dark energy can be discovered in the Lab through noise measurements of Josephson junctions is incorrect. In particular, they emphasize that the measured noise spectrum in Josephson junctions is a consequence of the fluctuation dissipation theorem, while our argument was based on equilibrium statistical mechanics. In this note we show that the fluctuation dissipation relation does not depend upon any shift of vacuum (zero-point) energies, and therefore, as already concluded in our previous paper, dark energy has nothing to do with the proposed measurements.
\end{abstract}

\section{Introduction}

It has been suggested by Beck and Mackey [1 that part of the zero-point energy of the radiation field that is gravitationally active can be determined from noise measurements of Josephson junctions. This caused some widespread attention. In a reaction we 2] we thought we had clearly shown that there is no basis for this claim, by following the reasoning in [1] for a much simpler model, for which it is very obvious that the authors misinterpreted their formulae. More generally, we stated that the absolute value of the zero-point energy of a quantum mechanical system has no physical meaning as long as gravitational coupling is ignored. All that is measurable are changes of the zero-point energy under variations of system parameters or of external couplings, like an applied voltage. 
Recently, Beck and Mackey argued [3] that our argument does not apply to their original proposal. They state in particular that the measured noise spectrum of Josephson junctions is a consequence of the fluctuationdissipation theorem, while our argument " is based on equilibrium statistical mechanics and does not incorporate non-equilibrium effects".

Mainly for this reason we demonstrate below explicitly that the fluctuationdissipation relation is immune to any shift of vacuum (zero-point) energies. In particular, the vacuum energy of the radiation field, cut-off at some frequency, does not enter in the fluctuation-dissipation theorem. The misinterpretation of the formulae by Beck and Mackey is, as we shall show, exactly of the same type we pointed out in our first reply [2].

\section{On the fluctuation-dissipation theorem}

For the sake of our argument, we have to briefly recall a derivation of the famous fluctuation-dissipation theorem, originally discovered by Callen and Welton [4].

Mathematically, the fluctuation-dissipation theorem relates for an operator $Q(t)=e^{i H t} Q e^{-i H t}$ ( $H$ the Hamiltonian of the system) the canonical expectation values (denoted by $\langle\cdot\rangle$ ) of the commutator $[Q(t), Q(0)]_{-}$and the anti-commutator $[Q(t), Q(0)]_{+}$.

Let $\alpha^{\prime \prime}(\omega)$ be the Fourier transform of $\frac{1}{2}\left\langle[Q(t), Q(0)]_{-}\right\rangle$,

$$
\alpha^{\prime \prime}(\omega)=\frac{1}{2} \int_{-\infty}^{\infty}\left\langle[Q(t), Q(0)]_{-}\right\rangle e^{i \omega t} d t
$$

In physical applications $\alpha^{\prime \prime}(\omega)$ is the imaginary part of a generalized susceptibility $\alpha(\omega)$ (Kubo formula for linear response), and describes energy dissipation caused by an external perturbation. It is now a matter of at most a few lines ${ }^{1}$ to show that

$$
\frac{1}{2}\left\langle[Q(t), Q(0)]_{+}\right\rangle=\frac{1}{\pi} \int_{0}^{\infty} \operatorname{coth} \frac{\beta \omega}{2} \alpha^{\prime \prime}(\omega) e^{-i \omega t} d \omega
$$

$(\beta=1 / k T, \hbar=1)$.

For $t=0$ this formula expresses the fluctuation $\left\langle Q^{2}\right\rangle$ in terms of the susceptibility. A slightly different form is

$$
\left\langle Q^{2}\right\rangle=\frac{2}{\pi} \int_{0}^{\infty}\left[\frac{1}{2} \omega+\frac{\omega}{e^{\beta \omega}-1}\right] \alpha^{\prime \prime}(\omega) \frac{d \omega}{\omega} .
$$

\footnotetext{
${ }^{1}$ All that one needs is that the Fourier transform of $\langle Q Q(t)\rangle$ is equal to that of $\langle Q(t) Q\rangle$ times $e^{-\beta \omega}, \beta=1 / k T, \hbar=1$.
} 
This connection is what one calls the fluctuation-dissipation theorem. The formula used by Beck and Mackey is equivalent to (3). They use the relation between $\left\langle\dot{Q}^{2}\right\rangle$ and $\alpha^{\prime \prime}(\omega)$, which is obtained from (3) by multiplying the integrand with $\omega^{2}$.

Obviously, $\left\langle[Q(t), Q(0)]_{ \pm}\right\rangle$do not change under the substitution $H \rightarrow$ $H+$ const. Therefore, this substitution does not induce an additive constant in the square bracket of (3), contrary to what was suggested by Beck and Mackey in their equation (5.13). The term $\frac{1}{2} \omega$ in the square bracket has nothing to do with the ground state energy of the system. The latter does not show up in the fluctuation-dissipation theorem, and can be treated as an arbitrary normalization.

Testing relations like (3) amounts to testing basic quantum theory, and has nothing to do with dark energy.

\section{Concluding remarks}

We hope to have shown in sufficient detail that vacuum (zero-point) energies do not show up in any application of the fluctuation-dissipation theorem. Therefore, the change suggested in eq. (5.13) of Ref. [3], is wrong. The term $\frac{1}{2} \omega$ in the square bracket of (3) has nothing to do with vacuum energies.

In their rebuttal [3], Beck and Mackey make in Sect. $V$ the following general statement: "The theory of dissipative non-equilibrium quantum systems, such as driven Josephson junctions, is much less well understood than the Casimir effect. Whether the dissipative quantum theory underlying resistivity shunted Josephson junctions can be renormalized is presently unclear. Hence the absolute value of the vacuum energy may well have physical meaning for these kinds of superconducting quantum systems." Our comment to this - beside our concrete remarks in the previous section - is: The basic theory underlying resistivity shunted Josephson is a renormalizable theory, namely quantum electrodynamics. In this theory the vacuum energy can be normalized to any value, without changing any observable prediction. This can only change when an enlarged theory containing gravity is considered. Outside gravity, vacuum energies are unmeasurable. (The standard model of particle physics is renormalizable.)

\section{References}

[1] C. Beck and M. C. Mackey, Phys. Lett. B 605, 295 (2005); astro-ph/0406504. 
[2] Ph. Jetzer and N. Straumann, Phys. Lett. B 606, 77 (2005); astro-ph/0411034.

[3] C. Beck and M. C. Mackey, astro-ph/0603397.

[4] H. Callen and T. Welton, Phys. Rev. 83, 34 (1951). 